\title{
Thioridazine Hydrochloride
}

National Cancer Institute

\section{Source}

National Cancer Institute. Thioridazine Hydrochloride. NCI Thesaurus. Code C29498.

The hydrochloride salt form of thioridazine, a piperidine phenothiazine derivative and a dopamine antagonist with antipsychotic property. Thioridazine hydrochloride binds to mesolimbic postsynaptic dopamine receptor D2, thereby decreasing dopamine activity leading to decreased psychotic effects, such as hallucinations and delusions. In addition, this agent binds to serotonin 5-HT2 receptors, resulting in decreased serotonin activity. 